\title{
The Methodology for Evaluating the Safety of Operations at the Uncontrolled Aerodromes
}

\author{
Vladimír Plos \\ Laboratory of Aviation Safety and Security, \\ Department of Air Transport, Faculty of Transportation \\ Sciences, Czech Technical University \\ Horská 3, Praha 2, 128 03, Czech Republic \\ e-mail: plosvlad@fd.cvut.cz
}

Peter Vittek

Laboratory of Aviation Safety and Security, Department of Air Transport, Faculty of Transportation Sciences, Czech Technical University Horská 3, Praha 2, 128 03, Czech Republic

\author{
Jakub Kraus \\ ATM Systems Laboratory, \\ Department of Air Transport, Faculty of Transportation \\ Sciences, Czech Technical University \\ Horská 3, Praha 2, 128 03, Czech Republic \\ e-mail: kraus@fd.cvut.cz \\ Tomáš Duša \\ Department of Air Transport, \\ Department of Air Transport, Faculty of Transportation \\ Sciences, Czech Technical University \\ Horská 3, Praha 2, 128 03, Czech Republic
}

\begin{abstract}
The objective of this methodology is to determine the general procedure for the evaluation of safety indicators for operation at uncontrolled aerodromes. The basis of this assessment is to determine the processes that take place at the aerodrome and finding critical points in these processes. They are determined on the basis of observations at aerodromes and are based on current legislation. The result of applying this methodology to aerodrome operations is the identification of several indicators, which monitoring and the adoption of safety measures can enhance safety at these uncontrolled aerodromes in the Czech Republic.
\end{abstract}

Keywords - safety, airport procedures, aviation safety, safety evaluation

\section{INTRODUCTION}

Operational safety at small aerodromes with uncontrolled operations in the Czech Republic is currently discussed topic. While safety of "large" commercial air transport is extensively regulated and controlled by the supervisory authorities, and has various system tools for safety monitoring in operation, "small" aviation is in this regard left behind, that's why there are not developed a breakthrough procedures for it and it uses only the gradual acceptance of best practices of commercial air transport, which after adjustment for small aviation can be applied in an environment of aero clubs and uncontrolled aerodromes.

Currently, small aviation is focused mainly to reactive approach safety. This approach consists of the aviation accidents investigation from general aviation and on the results of these investigations based adoption of various safety measures and safety training. Organization of such training is a good start, because the importance of knowledge gained over time, which leads to discussion of various events - incidents / accidents. This effort, however, must not remain isolated and needs to be further developed. This can be ensured by monitoring of events that are triggers of dangerous events. However, the actual occurrence of these phenomena may not even mean the realization of an incident / accident. This approach is based on the prof. James Reason model, who defined the incident / accident as a chain of events. If these events happened alone it does not mean the realization of an accident, but if there is a combination of more such events in the "right" order at a time, it results in realization of incident / accident.

\section{THE METHODOLOGY FOR CREATING OF SAFETY INDICATORS AT SMALL UNCONTROLLED AERODROMES}

This methodology is based on process modelling and finding critical points in these processes that may lead to the realization of events with safety implications. The critical points exist at every aerodrome and depend on an individual AFIS officers and pilots approach to individual situations.

The methodology considers the basic processes that need to be addressed. The modelling of these processes is based on observations at aerodromes and on the basis of the legislation for aviation in the Czech Republic. The processes were modelled for the basic operation from the engines start-up and aircraft taxiing over the area through the take-off and flight at aerodrome circuit to the landing and leaving the runway. Models also describe the arrival of foreign aircraft to the airspace around the aerodrome or aircraft departure from the airport.

The actual process of creating indicators is started by modelling of processes for the aerodrome with regard to the specific features of the aerodrome - geographic location, aerodrome layout, etc. the basis is a general description of the process of departure and arrival at the aerodrome, see Figure 1. 


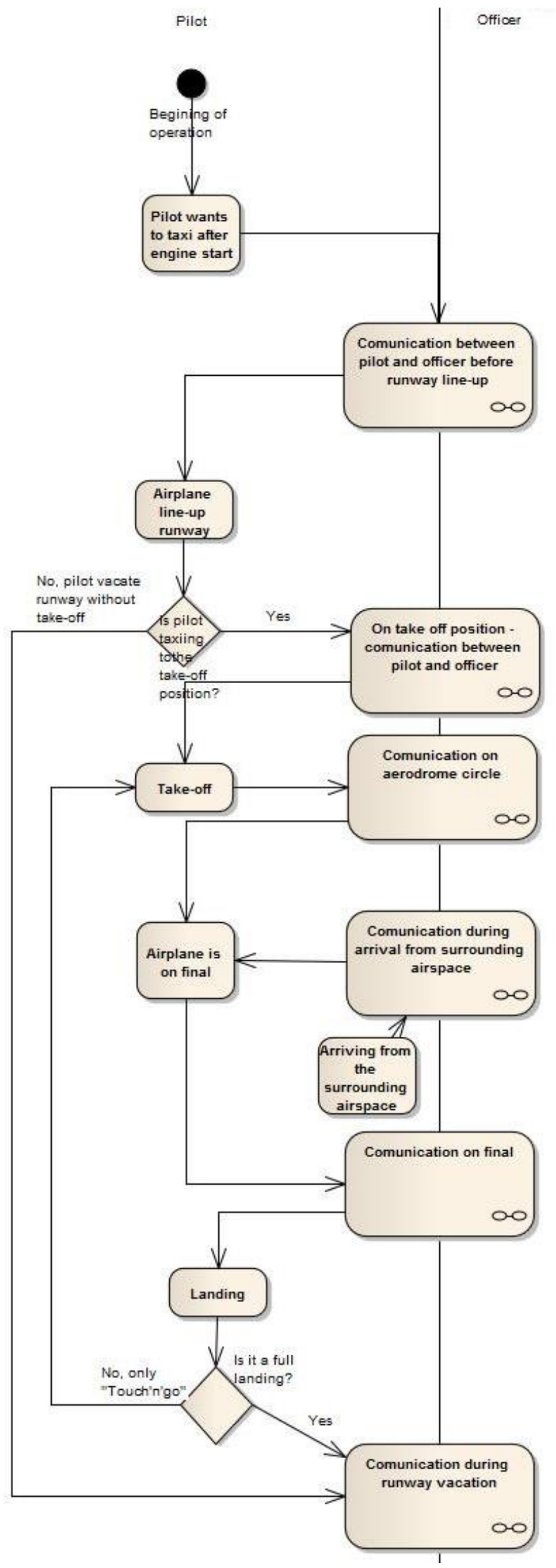

Figure 1. General process of departure and arrival at the aerodrome
Each process should be described in full detail; to have the distinctive level of individual tasks and operations as fine as possible, for searching of possible deviations. The operations and sub-processes are necessary to assign to the right stakeholder, which is in the process involved. This is very important for targeting safety measures.

Here follow some examples of detailed models of individual communication processes between AFIS and aircraft crew members.

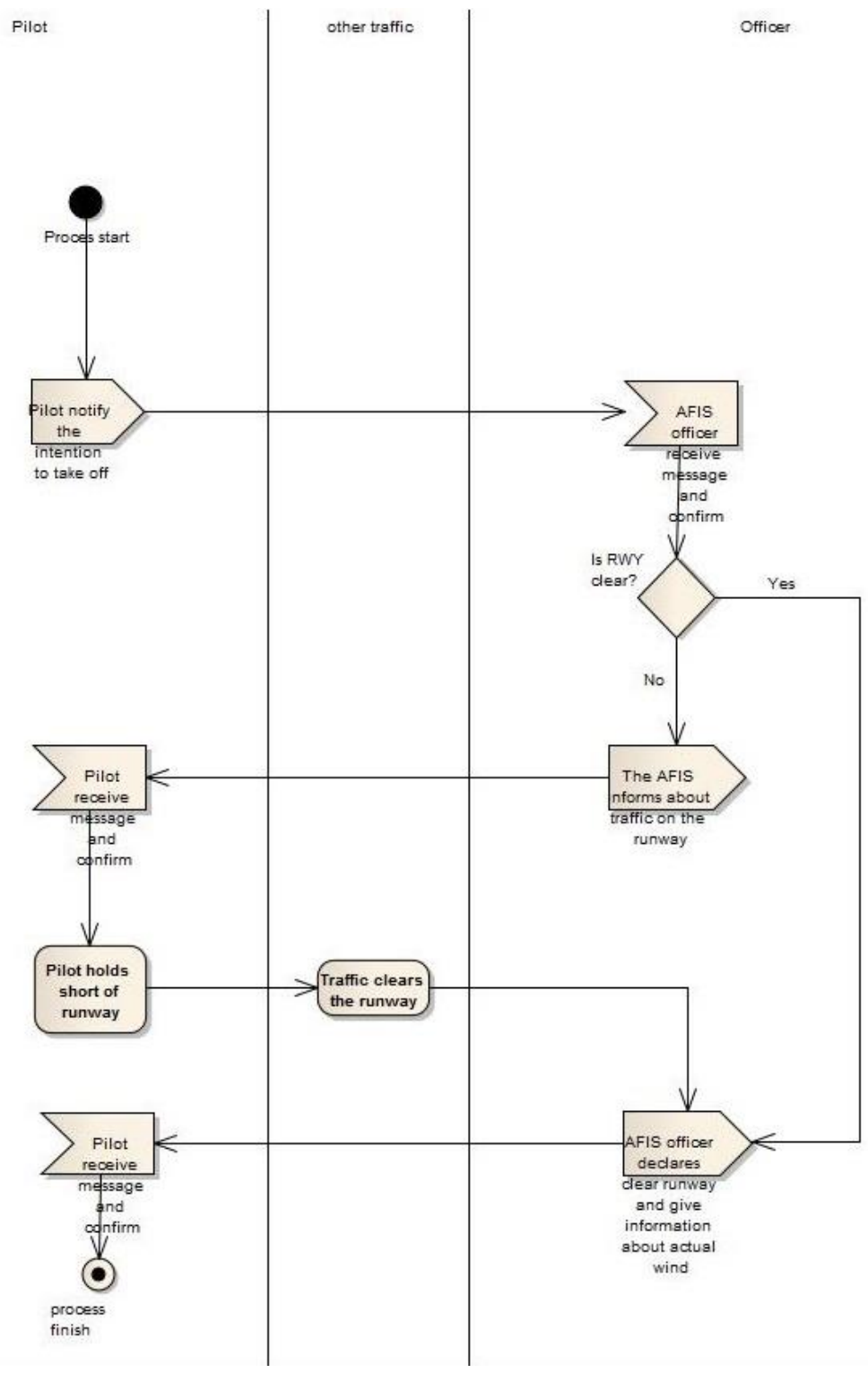

Figure 2. Process before take-off 


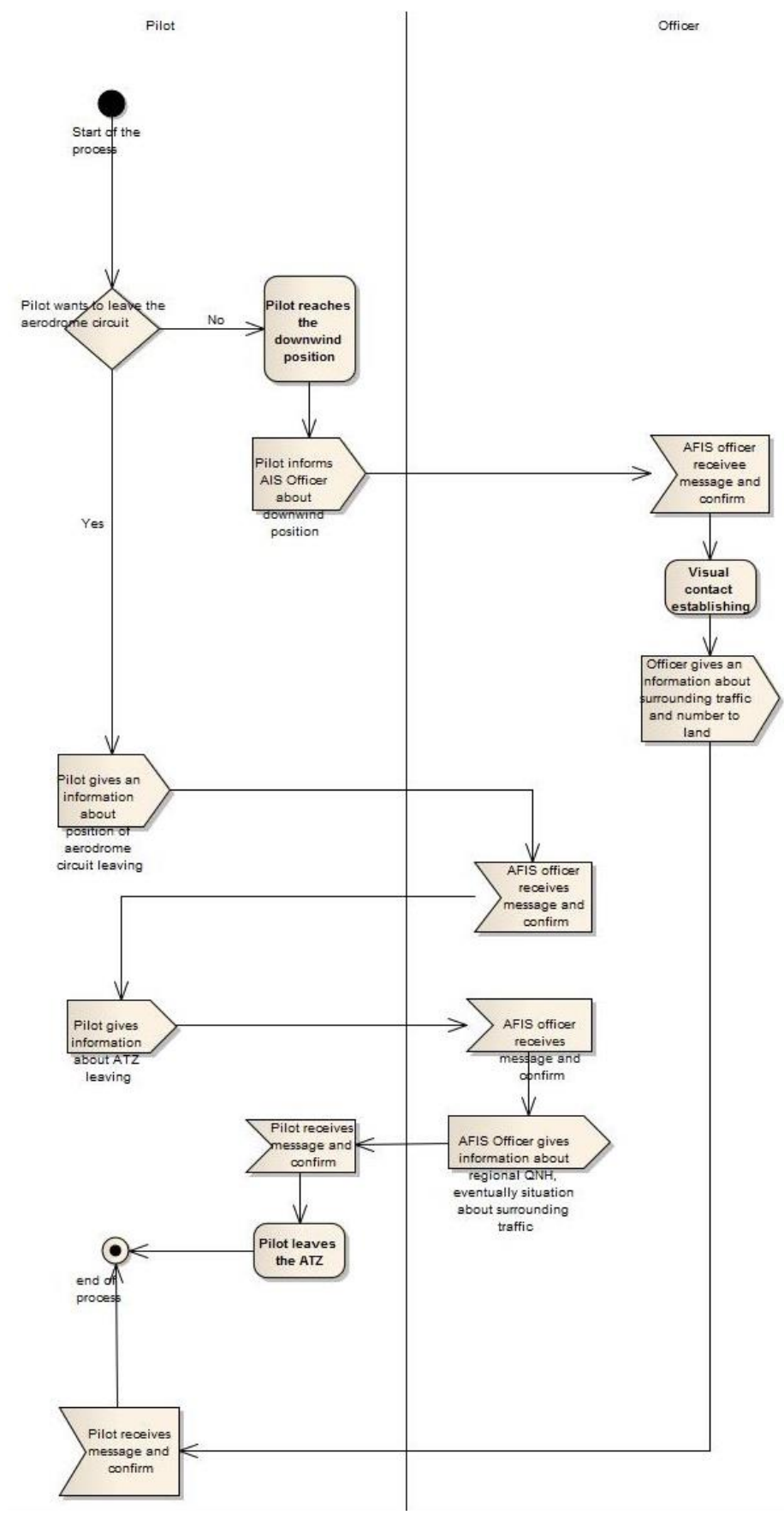

Figure 3. Process of the flying on aerodrome circuit

These models are intended to describe the ideal procedure. However, in these processes could occur partial errors and inaccuracies that can lead to the realization of an incident / accident. In such cases it is necessary to create models of the critical scenarios. To this, again, may serve some basic models based on the model of the process of departure and arrival at the aerodrome. These models show a deviation from standard procedures and the consequences that this deviation may have. The following is an example of such a crisis scenario modelled on the basis of possible traffic situations.

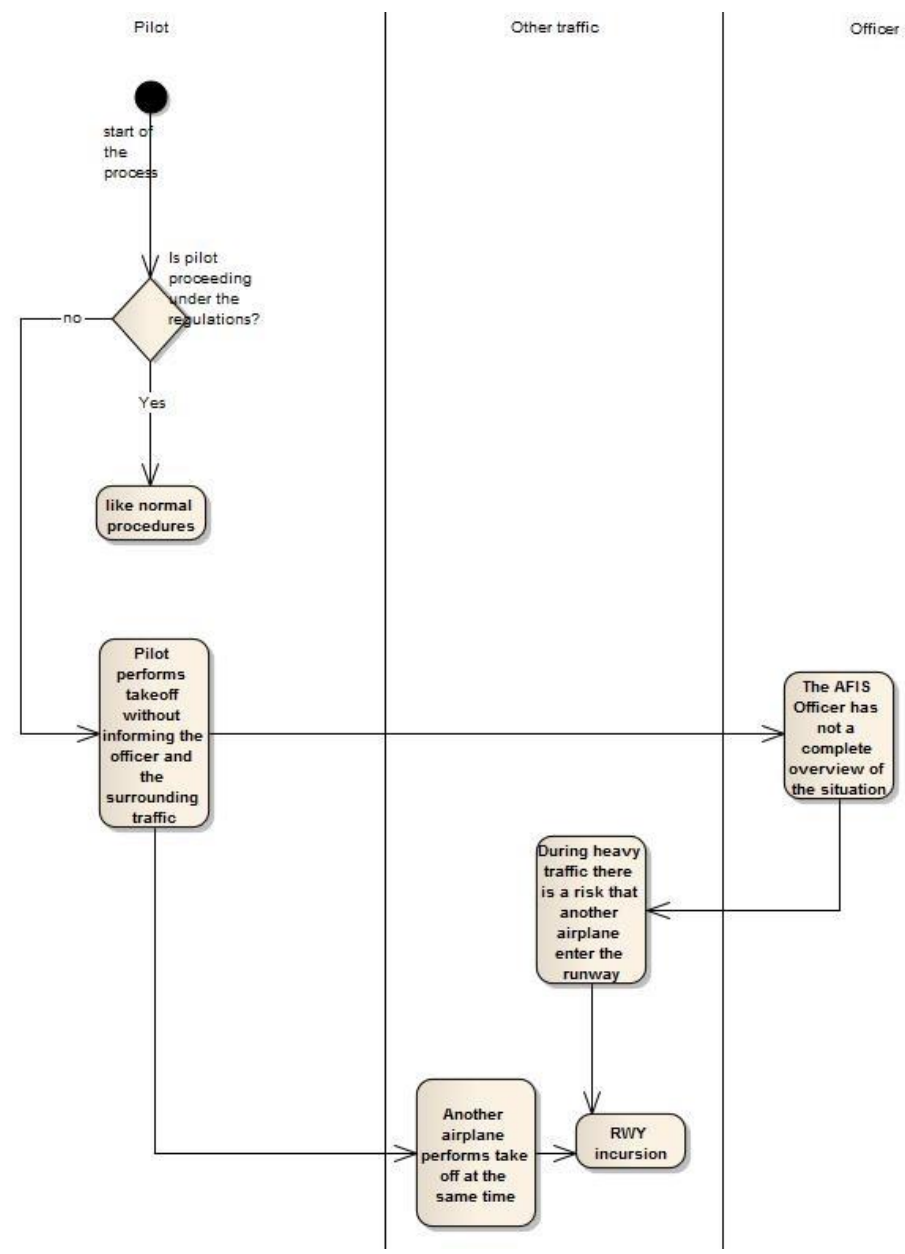

Figure 4. The process of take-off without informing the officer and the surrounding traffic

To obtain these crisis scenarios, we can use two approaches. The first one is the observation of the actual operation and recording the variation and potential consequences of these deviations. This method is reactive. The second one, far more progressive, is the method of creating scenarios based on hypothetical situations that may arise in a given situation. It is de facto a system of hazard identification, as described by SMS.

The hazard identification must not be limited just to saying "this process has the potential for the creation of dangerous situation", but this potential should be developed into a detailed analysis and into the possibility of critical operations - e.g. position reporting - a crisis scenario goes through the steps incorrect position reporting, the AFIS officer is not able to establish visual contact, situational awareness is lost, the issuance of incorrect information, it may lead to wrong number to land, the usage of Go-Around procedures, or realization of an accident.

This way obtained crisis scenarios, respectively events arose from the realization of these scenarios, should be evaluated in terms of frequency and severity of the consequences. This review is based on the methodology of safety management system, which uses alphanumeric risk assessment. 
The frequency is calculated as the number of realizations per unit. In this case, it is appropriate to determine this unit as the number of movements at the aerodrome, e.g. 100. One single level is important for successive comparisons between the aerodromes and the determination of the safety performance. For frequency is important to select the appropriate levels for the subsequent division into five categories according to safety management system. These categories reach from negligible occurrence to very frequent occurrence.

The severity of the consequences of implementation is also based on the methodology of safety management system where the severity is divided into five levels - from no effect on health and property to catastrophic consequences.

With the obtained alphanumeric evaluation it is possible to focus only on those operations that hide excessive risks and adopt targeted measures to mitigate safety risks of the relevant processes. This mitigation is done by strengthen one of three layers of defence. These layers of defence are training, legislation and technology. The first two can be considered as the soft layers which are not so demanding from the economical point of view, but also not as efficient as the latter layer - technology. This kind of defence against hazardous events is economically demanding, but brings the greatest successes.

\section{CONCLUSION}

In this article mentioned methodology was created based on the grant SGS13/090/OHK2/1T/16 which has investigated the issue of safety at uncontrolled aerodromes with a focus to the area of communication between the AFIS and aircraft crew. The officer at the aerodrome rely with some exceptions only on reports positions from aircraft crews and he is therefore exposed to the risk of submitting inaccurate information and subsequent poor evaluation of the situation resulting from the loss of awareness. Some means to increase safety would be to introduce a system of indicators to monitor the occurrence of events which may lead to realization of dangerous situation. These indicators are based on the process models, which was defined by the responsible person at each aerodrome from analysed results of the identification of critical processes. On these critical processes should be build emergency scenarios with assessment of the severity and frequency of implementation.

If the number of realizations of such events increase, to which will point the appropriate safety indicator, it would be possible to respond with targeted safety measures with options such as procedure amendment to the legislative basis or possibly the introduction of simple surveillance system for each aerodrome. Such a surveillance system would provide at least basic overview of air traffic moving around the aerodrome within ATZ to the officer.

\section{ACKNOWLEDGMENT}

This paper was supported by the Grant Agency of the Czech Technical University in Prague, grant No. SGS13/090/OHK2/1T/16.

\section{REFERENCES}

[1] L11 standard - ICAO annexes [online]. [cit. 2014-01-05]. Available at: <http://lis.rlp.cz/predpisy/predpisy/dokumenty/L/L11/data/print/L11_cely.pdf>

[2] ØIEN, K., I.B. UTNE a I.A. HERRERA. Building Safety indicators: Part 1 - Theoretical foundation. Safety Science. 2011, vol. 49, issue 2, p. 148 161. DOI: 10.1016/j.ssci.2010.05.012. available at: <http://linkinghub.elsevier.com/retrieve/pii/S0925753510001335>.

[3] ØIEN, K., I.B. UTNE, R.K. TINMANNSVIK a S. MASSAIU. Part 2 Application, practices and results. Safety Science. 2011, vol. 49, issue 2, s. 162-171. DOI: 10.1016/j.ssci.2010.05.015. Availabel at: http://linkinghub.elsevier.com/retrieve/pii/S0925753510001360

[4] Eurocontrol AFIS manual. [online]. Eurocontrol. Available at <http://www.skybrary.aero/bookshelf/books/1446.pdf> 